\title{
A Karaka Based Approach to Parsing of Indian Languages
}

\author{
Akshar Bharati Rajeev Sangal \\ Department of Computer Science and Engineering \\ Indian Institute of Technology Kanpur \\ Kanpur 208016 India \\ Abstract \\ A karaka based approich for patsing of tndiall laneuages is \\ described. It has been used for buildire a parser of Hindi for \\ $\Lambda$ lexicaliged examal formalism has beer developed that \\ allows constraints to be apecified between 'demand' and 'source' \\ has two important novel features: (i) It has a local word
} a prototype Machine Translation system. words (e.g., between verb ard its karaka rojes). The parser. grouping phase in which word groups are fotmed using local' information only. They are formed based on finitestate machine specifications thus resulting in a fast grouper. (ii) The parsex. is a general constrait solvel. It firat transforms the constraints to an integer programing problem and then solves it.

1. Introduction

Languages belonging to the fudian linguistic area share several common features. They are relatively word order free, nominals are inflected or have post position case matkers (colloctively called as having vibhakti), have verb complexes consisting of sequences of verbs (possibly joined together into a single word), etc. There are also commonalities in vocabulory, in senses spanned by a word in one language to those of its counterpartin another Indian language, oto.

We base our grammar on the karaka (pronounced kaarak) structure. It is necessary to mention that although karakas are thought of as similar to rases, thay are fundamentally different: "The pivotal cateeories of tho abstract syntactio representalion are the karakas, the gramatical functions as. signed to rominals in relation to the verbal root. They are neither semantic nor morphological categories in themselveg but correspond to semantics accordine to rules specified in the grammar and to morphology according to other rulers specified in the grammar" [Kiparsky, 82].

Before describing our crammar formalistil, let us look at the parser struc

\section{ture:}

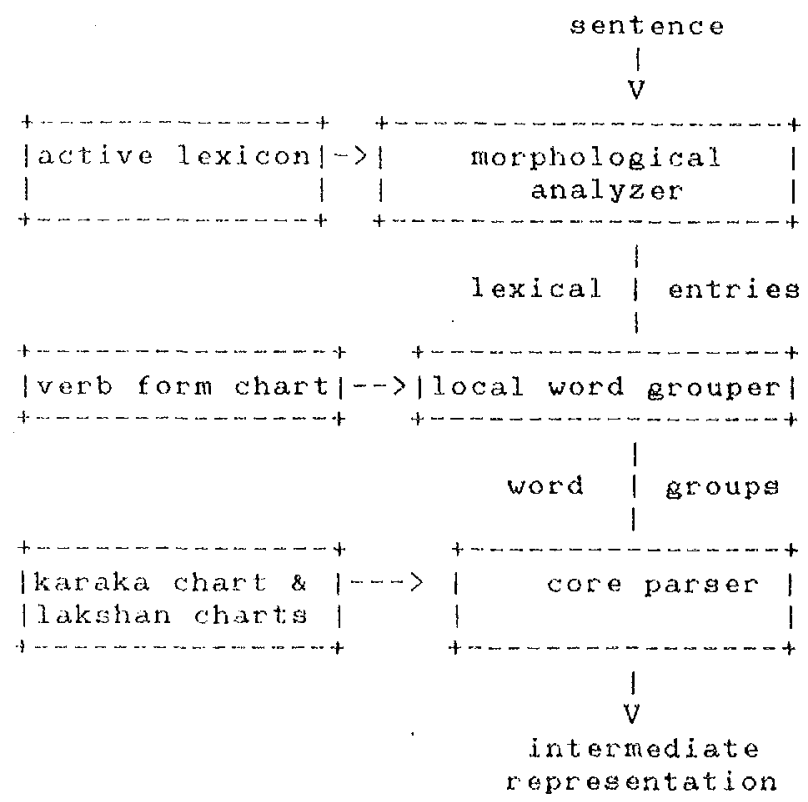

Function of the morphological analyzer is to take each word in the input sontence and extract its root and other associated eranmatical information. This information forms the irsut to the local word erouper (LWG). 


\section{Local Word Grouper (LWG)}

The function of this block is to form the word groups on the basis of the '10cal information' (i.e., information based on adjacent words) which will need no revision later on. This implies that whenever there is a possibility of more than one grouping for some word, they will not be grouped together by the LWG.

This block has been introduced to reduce the load on the core parser resulting in increased efficiency and simplicity of the overall systen.

The following example illustrates the job done by the LWG. In the following sentence in Hindi:

ladake adhyapak ko haar pahana rahe hein लउतके उत्यापक को हार पहना रे है। boys teacher to garland garland - ing

(Boys are garlanding the teacher.)

the output correspondine to the word 'ladake' forms one unit, words 'adhyapak' and 'ko' form the next unit, sinilarly 'pahana', 'sahe' and 'hein' will form the last unit.

\section{Core Parses}

The function of the core parser is to accept the input from l.wG and produce an 'intermediate language' representation (i.e parsed structure along with the identified karakd roles) of the given source language sentence. The core parser has to perform essentially two kinds of tasks

1) karaka role assignment for verbs

2) sense disambiguation for verbs and nouns respectively.

For translating arrong Yinian latiguages, assignment of karaka roles is sufficient. one need not do the semantic role assignment after the karaka assienment.

Let us now look at the grammar.

\subsection{Grammar Formal ism}

The notion of karaka* velation is * Here, we uge the word 'karaka' in an ax tended serse which includes hetu', 'tadarthya" etc. in addition to actual karaw kas. central to the model. These are semantico-syntactic relations between the verb(s) and the rominals in a sentence. The computational grammar specifies a napping from the nominalg and the verb(g) in a sertence to karaka relations between them. Similarly, other rules of gramar provide a mapping from karaka relations to (deep) semantic relations between the verb(s) and the nominals. Thus, the karaka relations by themselves do not aive the semantics. They specify relations which mediate between vibhakti of nominala and verb form on one hand and gemantic relations on the other [Bharati, Chaitanya, Sangal, 90].

For each verb, for one of its forms called as basic, there is a default karaka chart. The default karak chart specifies a mapping from vibhaktig to karakas when that verb-form is uaed in a sentence. (Karaka chart has additional information besides vibhakti pertaining to 'yogyata' of the nominals. This serves to reduce the possible parses. Yogyata gives the semantic type that must be satisfied by the word group that serves in the karaka role.)

When a verb-form other than the basic occurs in a sentence, the applicable karaka chart is obtained by taking the default karaka chart and transforming it using the verb type and its form. The new karaka chart defines the mapping from vibhakti to karaka relations for the sentence. Thus, for example, jotata hai, (ploughs) in A. 1 has the default karaka chart which says that karta takes no parsarg (Ram). However, for 'jota' (ploughed) in A.2, or A.4, the karaka chart is transformed so that the karta takes the vibhakti 'ne' "ko' or 'se?",

A.1 Ram khet ko jotata hai राम खेत को ओतता है।
Ram karm ko-parsarg plough -s. (Ram ploughs his farm.)

A.2 Ram ne khet ko jota. राम ने खेत को जोता ।

Rarn tre- farm ko- ploughed. (Ram ploughed the farm.)

A. 3 Ram ko khet jotana pada. राम को इेत जोतना पडा । Ram ko- farm plough had-to. (Ram had to ploweh the farm.) 


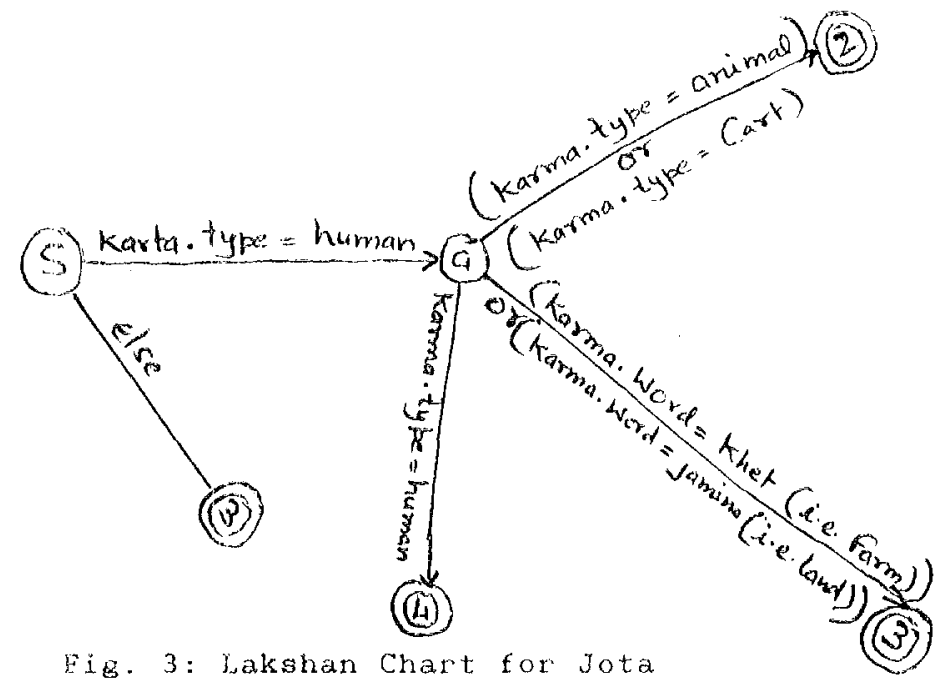

Finally, besides the merged karaka charts associated with individual verbs, there is also a global table of common karakas. It pertains to adhikatana karaka (time and place), hetu (cause), etce and is applicable to all the verbs. It can be used to account for source word groups that romain after satiofying the mandatory karakas. In this sense, it only contains optional karakas.

\section{3 parsing}

For the task of karaka asgienment. the core parser uses the fundamental principle of 'akanksha' (demand unit) and 'yogyata' (qualification of the source unit).

The linguistic units which play the role of demand and source word groups can vary depending on the parse cycle. In the case of sinfle sentences, only one cycle is needed in which verb groups and sone special rour groups (e.6. "paas'(near), "door'(fat) ete.) play the role of demand word groups, and nour Broups ard predicative adjectives play the role of source word groups.

Durine the parsing process, each of the source word groups may be tested against each of the karaka festrictions in each of the kizraka charts of the demand word eroups. An appropriate data siructure may be created storing the source word groups and the karak restrictions (in karaka charts of derand groups) they satisfy. We call each such entry as a candidate variable.
Typicaliy, a number of gource word groups will qualify for a particular defand. The job of the core paxser is to make an appropriate assignIllent of the candidates, subject to cer tain constraints such as the following:

1) one candidate source word group cannot satisfy more than one demand of the same demand word.

2) every obligatory demand mugt be satigfied in some karaka chart of every demand word group.

3) every source word must have an assigninent.

4) if more than one interpretation of a source word is available, then exactly one has to be selected.

The above problem is transformed to ar integer programming problem. Assigning 1 to a candidate variable means that the particular karaka relation between the source word group and the demand word group holds; 0 stands for otherwise. All the various types of constraints mentioned above can be specified in a very natural manner using algebraic inequali$t i$ es in integer programming. Having a set of candidate variables assigned to 1 not only identifies the karaka relations which can be used to zet the deep cases, but also identifies the karaka chart which serves to identify the senge of the verb group, ets.

Moreover Integer programming algo permits a linguist to express preferences amons various candidates for a particular dernand. A typical example of such a preference can be iven. For example, for most of the verbs an animate thing is more likely to be the karta thar induinate things, and among aninates human beings are more likely candidates to b.: karta than non-human candidates. These preferences would simply order the multiple parses if any in the absence of other information.

The parsing strategy actually adopted in the system makes use of the merged karaka char $t$ and corresponds to AnvitAbhidhanvad, a theory of mimama school of the Indiar gramatical tradition. In this approach, we first determine the karaka relationships amore the demand ard cource word groups. (These are determined 
A. 4 Ram se khet nahi jota gaya.

राम से खेत नही जोता गया।

Ram se- Earm not plough could.

(Ram could not plough the farm.)

The above principle allows us to deal with active passives. The verb forms for active and passive are just two special cases of the forms a verb can take.

For example, the verb 'jota' in Hindi has four different meanings listed in the dictionary:

1) harness (e.g., Ram ne bail ko kolhu me jota, or Ram harnessed the bullock for (turning) the crusher)

शाम ने बेल को कोट्ह में जोता ।

2) hitching the cart (e.g., Ram ne gaadi ko jota, or Ram hitched the cart.) राम ने गाडी को जोता।

3) plough (e.e., Ram ne janindar ka khet jota, or Ram ploughed the landlord's farm.') राम ने जमींदार का इसेत जोता ।

4) exploit (e.g., Ram ne naukar ko kaam me jota diya, or Ram exploited his servant by putting him to (hard) work.) राम ने नौकर को काम में जोत दिया

For earh of the four senses, a karaka chart can be created. A karaka chart specifies the mandatory karakas (i.e., which must be filled for the senterse to be grammatical), optional karakas, and desirable karakas. For each of the karakas, it specifies the vibhakti (i.e., inflection or post position marker), and the semantic specification (typically in the form of semantic type) to be satisfied by the source word (eroup). Such a specification for a karaka in a karaka chart is called a karaka restriction. Thus, the karaka chart for the 'hitching' sense of 'jota' has two mandatory karaka restrictions: one for karta karaka (pronounced kartaa kaarak) and the other for karma karaka (pronounced karm kaarak). The former karaka relation maps to agent and the latter to patient semantic relation. As shown in Eig. 1, the restriction for karta karaka says that a source word group satisfying it must be present in the sentence, its vibhakti must be 0 , and its semantic type should be human.

restriction on karta karaka: karaka: karta mandatory: yes

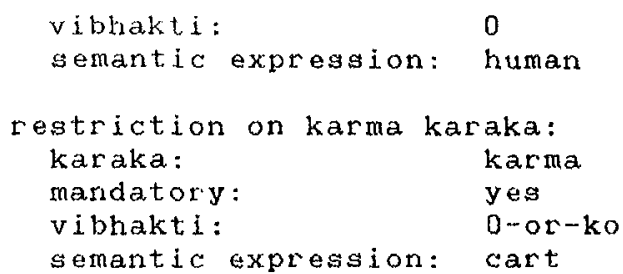

Fig. 1: Karaka Chart for Jota (Sense 2)

\subsection{Refinitg the Grammar Model}

The actual erammar we use in the system is based on the model discusaed above. However, it differs from it slightly so as to have a fagter paraer.

Iristead of a separate karaka chart for each genge of a verb, we have a gingle mereed karaka chert. It conglgts of a set of karaka regtrictions where a restriction for a particular karaka relation is obtained by taking the logical-or of the necessary vibhakti and semantic typea for the same karaka relation in the different karaka chart.s. For examplo, semantic type in reatriction for karma karaka for the merged karaka chart is obtained by taking logical-or of semantic types in karma karaka restrictiong in the different karaka charta. Eig. 2 ghows the merged karaka chart for jota.

Karaka Necessity Vibhakti Semantic Type

$\begin{array}{llll}\text { karta } & m & 0 & \text { animate } \\ \text { karma } & m & 0-k 0 & \begin{array}{l}\text { animate or } \\ \text { ingtrument or } \\ \text { land }\end{array} \\ \text { karana } & d & \text { se-dvara animate or } \\ & & \text { ingtrument }\end{array}$

Eig. 2: Merged Karaka Chart for Jota

As the separate karaka charta are no longer available for distinguishing among the senses of the main verb, separate information is needed. This information is available in the form of lakshan charts or discrimination nets. Thea neta can be obtained by looking at the separate karaka charts and identifying features that help us in distinguishing among the different senses. An example lakshan chart for jota is given in Fig. 3 . 
by tosting the source word eroups adecinst. karaka restrictions in the luered karaka chart, and then oolvolug the integm prom gramanter problem.) The word meaning is deternined only later whing the latishan charts on the karaka assignment.

1. Cotsciusions

The majot featutes of out apploath can be summatized as follows:

1) a parsing etrategy based on 'akarksha' (demand) and yogyata' (qualification of the source unit). Note that he karaka charts expressing restrictions els above are on inar to wabcategorization and selectional resitlictions, but are sot identical to them. Sub categoriation refers to ceep cases, and selectional restrico tiong usually specify genantic types. Here we use karaka relations, and specify not just semantic types but also post - position markers. It should, of course, be noted that these ideas play a central role in our eramar and parser.

2) parsing strategy that uses nergod faraka chat to do kataka assignnent, cond only later does the serise selection for nouns and verbs usine lakshan charts.

3) formulation of the core parsing problen as integer programing problem. It should be noted that integer programmirg is a general purpose technique making a large amount of towet and flexibijity available to the parser. This is at the cost of efficiency if the rutuber of variables to be hand ed imultaneously is large (though our current parser funs fairly fast). We are engaged in building a special constraint solver that will use this power ondy when necessary [Ramesh, gu].

The gramar and the parser described above are part of a machine translation system for Indian lareuages based on an interlingua [Sangal \& Chaitnya, 87]. Generator in the system uas the same erammar. In principle, each of the stages of the parser is reversed [Sencupta, 89].
Acknowloderent:

We wuld like to acknowledge the prinsipal source of ideas in this paper: Dr. Vireet chat anya.

Reforences

[Bharati, Chaitanya \& Sareal, 90] A Computatiotial Grammar for Indian

Larguage processing, A. Bharati, $y$. Cha itanya, and R. Sangal, Technical Report IRCS-90-96, Dept. of Computer Sc. \& Enge., 1.X.T. Karpur, 1990

[Kiparsky,92] Some Theoretical probleme in Punini's Grammar, P. Kiparsky, Bhandarkar Oriental Research institute, Pure, 1982

[Ramesh, 90] Constraints in Logic Pro araming, R.V. Ramesh, M.Tech. thesis, Dept. of Computer Sc. Enge, I.1.T. Kanpur, Mar. 1990.

[Sangal \& Chaitanya, 87] An Intermediate Languege for Machine Translation: An Approach based on Sangkrit uging Conceptual Graph Notation, Computer Science \& Informatics, J. of Computer Society of India, 17, 1, pp. 921, 1987.

[Sangal, Chajtanya \& Karnick, 88] An Approach to Machine Tramslation in Indian Languages, Proc. of Indo-us Workshop on Systems and Signal Processing, Indian Institute of $\mathrm{Sci}-$ ence, Bangalore, Jan. 1988.

[Sen Gupta, 89] Some Aspects of Language Generation, Rimli Sen Gupta, M.Tech. thesis, Dept. of Electrical Enge, I.I.T. Kanpur, 1989. 